ly damaging have a much lower growth rate. The extent of the damage to normal tissue may therefore not just be determined by the growth rate of a malignancy but also by the degree of general specificity of the substances produced by the tumour.

This model therefore seems capable of explaining some of the clinical manifestations of cancer. It suggests that malignant cells reduce the rate of growth and division of normal cells by producing substances which themselves play a normal part in the regulation of cell growth. It follows that removal or inhibition of these substances might convert a malignancy into the equivalent of a benign tumour, prevent the replacement of normal tissue and hence influence the course of the disease.

ERIKA COWARD

30 Langton Road,

London NW2 6QA, UK

\section{Limits on the selfishness of DNA}

SIR - Doolittle, Kirkwood and Dempster recently argued (Nature 307, 501; 1984) that some "selfish" DNAs (for example, elements which transpose duplicatively) may restrain their own reproduction within genomes to avoid driving their hosts, and hence themselves, into extinction. They suggested that such self-restraint should be expected to evolve in elements that impose a cost on their host's fitness which increases with copy number. That is, copy number should be limited so that host mortality does not exceed host reproduction. This view is fatally flawed, as it ignores a fundamental constraint on the evolution of "selfish" DNA.

Doolittle et al. presented a mathematical model which showed that a self-restrained element would be favoured over one with unlimited reproduction. They apparently did not realize that a potential host lacking either element would be favoured over hosts carrying one of the elements. An element which imposes any cost may be treated simply as a deleterious host gene, so selection of the host will eliminate that element. To persist, "selfish" DNA which is confined to one host (their model did not allow for transfer of the elements between hosts) must be beneficial to its host or be continuously generated de novo at a rate as great or greater than that of its loss due to selection (against its host) or vegetative segregation (failure to be vertically transmitted). The conclusion of Doolittle et al. may be reinterpreted to mean that self-restrained elements will be eliminated more slowly than unrestrained ones, but both will be eliminated. A different approach might show that self-restraint can evolve to avoid imposing any cost on the host, but modelling such a process might be difficult.

It has become common for biologists to imagine "selfish" DNA as a "genomic parasite", implying that it imposes a cost on its host, but most do not realize that true parasitism would require a mechanism for horizontal transfer. Genetic elements which can be transferred horizontally may be able to offset a cost to their hosts by colonizing new hosts. This would require a high enough transfer rate to balance their losses by vegetative segregation and selection against their hosts. Temperate bacteriophage, some plasmids and temperate-like viruses of eukaryotes may fall into this category, but one would have to know rates of transfer and intensities of selection to make such a claim. A generalization is clear - one should expect genetic elements without a mode of horizontal transfer to be beneficial to their hosts and/or continuously generated de novo at rates sufficiently high to make up for their losses.

RALPH V. EVANS

Department of Zoology,

University of Massachusetts,

Amherst, Massachusetts 01003, USA

\section{Earthquake prediction from $b$-values}

SIR - In 1981 W.D. Smith ${ }^{1}$ made determinations of the $b$-value for a series of "moving windows" for various areas in New Zealand. The " $b$-value" is the slope (best estimate) of the relationship between magnitude and frequency of earthquakes over a fixed interval and fixed area; a "moving window" comprises a fixed number of events, say from event 1 to 50 , the window being "moved" by shifting 10 events each time. Smith found that $b$-value maxima were associated with subsequent major earthquake events in accordance with a relationship he determined.

In 1982 , with a postgraduate student whose supervision had been entrusted to me, Smith's principles were applied to Papua New Guinea. Excellent records of earthquake events are kept by the Papua New Guinea Geological Survey and were made available to us. Over a series of five years, it was possible to isolate the earthquake events associated with certain tectonic features and for some of these, to apply Smith's method. We found that the relationship he devised was similarly close for Papua New Guinea earthquakes.

This may be of interest to anyone wishing to study $b$-value maxima as earthquake precursors. The work is recorded in a university report ${ }^{2}$. However, since the data covered 1,669 earthquake events, it was not practicable to publish it. It is not possible to predict earthquakes by this method from our work since the relationship is one between time after the $b$-value maximum and the magnitude of the subsequent event - the longer the time lapse, the greater the earthquake which follows. Furthermore, since then, Smith has had considerable doubts about the accuracy of the relationship he found (W.D. Smith, personal communication). However, it would be a pity if the data and findings, involving a considerable amount of work, should be unavailable to workers in the field, its only record being in a form relatively obscure to library searchers.

S. NOEL KIEK

145 Beach $R d$,

\section{Batehaven,}

New South Wales, Australia 2536

1. Smith, W.D. Nature 189, 135-139 (1981).

2. Emande, F.T. \& Kiek, S.N. Earthquake Precursors. Applications in Papua New Guinea (Papua New Guinea University of Technology, Lac, 1982).

\section{Brain, behaviour and the immune system}

SIR - We read with great interest the News and Views article on "Psychoimmunology before its time"' (Nature 309, 400; 1984), which calls attention to the now substantial evidence that brain and behavioural states can alter aspects of immune function. Attention is also called to other studies which have found links between similar behavioural states and health outcome. There has been a tendency, as noted in the article, to treat these two areas of research as if they were one and to confuse biological and epidemiological findings. Nature's near-scepticism concerning the explanatory power of the reported immune findings in relation to the role of stress in health outcome is well taken. But such an attitude might also be interpreted to suggest a sceptical approach to the biological findings themselves when they cannot adequately explain the epidemiological, such as with studies of bereavement. This type of reasoning would suffer from the same fault as that ascribed in the Nature article to the "psychoimmunologists" and further underscores the need to consider studies of behaviour and immune interactions in a biological context.

Current research on the effects of brain and behaviour on the immune system has provided reproducible, statistically and biologically significant data. The expanding knowledge about lymphocyte function and the developing technology may well provide the opportunity to elucidate further the association between brain and behavioural states and immunity. It remains to be determined whether such biological processes can explain aspects of physical illness.

STEVEN J. SCHLEIFER

STEVEN E. KELlER

MARVIN STEIN

Department of Psychiatry,

Mount Sinai Medical Center,

New York, New York 10029, USA

\section{Somatic gene mutation and} breast carcinoma (correction)

DUE to an editorial error, the above item of scientific correspondence (Nature 25 July, $310,103-104 ; 1984$ ) was incorrectly credited to a single author. It was in fact from M.A. Hulten, C.S. Rodgers and S.M. Hill, all at the Regional Cytogenetics Laboratory, East Birmingham Hospital, Birmingham B9 5ST, UK. 